\title{
Interleukin 13 on Microglia is Neurotoxic in Lipopolysaccharide-injected Striatum in vivo
}

\author{
Ah Reum Hong ${ }^{1 \dagger}$, Jae Geun Jang ${ }^{1 \dagger}$, Young Cheul Chung ${ }^{2 \dagger}$, So-Yoon Won ${ }^{3 *}$ and Byung Kwan Jin ${ }^{1,3 *}$ \\ ${ }^{1}$ Department of Neuroscience, Graduate School, School of Medicine, Kyung Hee University, Seoul 02447, \\ ${ }^{2}$ Department of Predictive Toxicology, Korea Institute of Toxicology, Daejeon 34114, \\ ${ }^{3}$ Department of Biochemistry \& Molecular Biology, School of Medicine, Kyung Hee University, Seoul 02447, Korea
}

\begin{abstract}
To explore the potential function of interleukin-13 (IL-13), lipopolysaccharide (LPS) or PBS as a control was unilaterally microinjected into striatum of rat brain. Seven days after LPS injection, there was a significant loss of neurons and microglial activation in the striatum, visualized by immunohistochemical staining against neuronal nuclei (NeuN) and the OX-42 (complement receptor type 3, CR3), respectively. In parallel, IL-13 immunoreactivity was increased as early as 3 days and sustained up to 7 days post LPS injection, compared to PBS-injected control and detected exclusively within microglia. Moreover, GFAP immunostaining and blood brain barrier (BBB) permeability evaluation showed the loss of astrocytes and disruption of BBB, respectively. By contrast, treatment with IL-13 neutralizing antibody (IL-13NA) protects NeuN ${ }^{+}$neurons against LPSinduced neurotoxicity in vivo. Accompanying neuroprotection, IL-13NA reduced loss of GFAP ${ }^{+}$astrocytes and damage of BBB in LPS-injected striatum. Intriguingly, treatment with IL-13NA produced neurotrophic factors (NTFs) on survived astrocytes in LPS-injected rat striatum. Taken together, the present study suggests that LPS induces expression of IL-13 on microglia, which contributes to neurodegeneration via damage on astrocytes and BBB disruption in the striatum in vivo.
\end{abstract}

Key words: Interleukin 13, Microglia, Neurodegeneration, Astrocytes, Neurotrophic factor, Striatum

\section{INTRODUCTION}

Neuroinflammation is a response produced by the activation of the brain's immune system due to various causes ranging from infection to autoimmunity. Among the immune cells in the brain are microglia, considering as resident macrophage [1]. Accumulating evidence has demonstrated that reactive microglia/macrophage play a critical role in the cause and progression of cell death in various neurodegenerative diseases including Parkinson's disease

\footnotetext{
Submitted October 1, 2021, Revised February 9, 2022,

Accepted February 16, 2022

* To whom correspondence should be addressed.

So-Yoon Won, TEL: 82-2-961-9288, FAX: 82-2-969-4570,

e-mail: sywon@khu.ac.kr

Byung Kwan Jin, TEL: 82-2-961-9288, FAX: 82-2-969-4570,

e-mail:bkjin@khu.ac.kr

${ }^{\dagger}$ These authors contributed equally to this article.
}

(PD), Alzheimer's disease (AD) and stroke [2,3]. Reactive microglia/macrophage exhibit large cell bodies with short, thick or no processes and exert neurotoxic effects by producing inflammatory cytokines, which consequently triggers severe neuroinflammation [4].

Astrocytes are the most abundant glial cells in the brain and exert neuroprotection by producing various neurotrophic factors such as ciliary neurotrophic factor (CNTF) $[5,6]$, mesencephalic astrocyte-derived neurotrophic factor (MANF) [7] and glial cell linederived neurotrophic factor (GDNF) [8]. Astrocytes are required to maintain the blood brain barrier (BBB) integrity [9], which was destructed in neurodegenerative diseases [10]. BBB protects the brain from potential neurotoxic molecules in circulation because the BBB helps regulate ion balance, nutrient transport, the entry of plasma components and blood cells $[9,11]$. Many studies including ours showed that $\mathrm{BBB}$ damage contributes to neurodegeneration in the striatum of 3-NP-treated mice [12] or 6-OHDA-treated 
rats [13], and in the substantia nigra of lipopolysaccharide (LPS)treated rats [14].

Interleukin-13 (IL-13) is well known anti-inflammatory cytokine and has diverse immunomodulatory functions on cell repair and regeneration in the brain $[15,16]$. IL-13 is expressed in reactive microglia in the brain and can produce both beneficial and harmful effects on neurons in vivo and in vitro [4, 17-19]. IL-13 increases oxidative stress, resulting in neurodegeneration in the hippocampus of amyloid beta $(A \beta)_{1-42}[20]$ or prothrombin kringle-2 (pKr-2)-treated rats [4] and in the substantia nigra of LPStreated mice [21]. On the other hand, IL-13 controls neuroinflammation and contributes to neuronal survival in LPS-treated rat cortex [22], in the pMCAo mouse model of cerebral ischemia [23] and in mouse model of multiple sclerosis [24].

Here we report that in LPS-injected rat striatum, IL-13 endogenously expressed in reactive microglia/macrophage contributes to degeneration of striatal neurons by destructing BBB integrity and astrocytes and inhibiting expression of neurotrophic factors on astrocytes such as CNTF and conserved dopamine neurotrophic factor (CDNF). The present data suggest that IL-13 is harmful to striatal neurons of LPS-lesioned rat in vivo.

\section{MATERIALS AND METHODS}

\section{Animals and stereotaxic surgery}

All surgical experiments were performed following the approved animal protocols and guidelines established by Committee on Animal Research of Kyung Hee University (KHSASP-21-364) to minimize suffering and the number of animals. Female SpragueDawley (SD) rats (230-260 g, purchased from Daehan Biolink, introduced from Taconic Co., USA) were housed under a 12:12 hr light: dark cycle at an ambient temperature of $21 \sim 23^{\circ} \mathrm{C}$. Throughout animal housing and the experiment, water and rat chow were available ad libitum.

Stereotaxic surgery was carried out as described [5]. SD rats anesthetized by intraperitoneally injection of chloral hydrate $(360 \mathrm{mg} /$ $\mathrm{kg}$, intraperitoneal injection) received unilateral administration of LPS (5 $\mu$ g in $3 \mu$ l Dulbeccós phosphate-buffered saline (PBS)) into the right striatum [anteroposterior (AP) $+0.7 \mathrm{~mm}$, mediolateral (ML) - $2.8 \mathrm{~mm}$, and dorsoventral (DV) - $5.0 \mathrm{~mm}$ from bregma], according to the atlas of Paxinos and Watson (Paxinos, 1998, The Rat Brain in Stereotaxic Coordinates 6th Edition). Injections of LPS or PBS or rat recombinant IL-13 (IL-13 (50 ng/3 $\mu$ ) / (300 $\mathrm{ng} / 3 \mu \mathrm{l}) ; \mathrm{R} \& \mathrm{D}$ Systems) as a control were made using a Hamilton syringe equipped with a $30 \mathrm{~S}$-gauge beveled needle and attached to a syringe pump (KD Scientific, MA, USA), at a rate of $1 \mu \mathrm{l} /$ $\min$. To neutralize function of IL-13, some animals stereotaxically received LPS with anti-murine IL-13-neutralizing antibody (IL13NA; $1 \mu \mathrm{g} / \mu \mathrm{l}$; R\&D Systems). IL-13NA alone or nonspecific goat IgG alone (goat IgG; $1 \mu \mathrm{g} / \mu \mathrm{l} ; \mathrm{R} \& \mathrm{D}$ Systems) as controls was also administered $[4,20,25]$. At the completion of each injection, the needle was left in place for $10 \mathrm{~min}$ and then slowly withdrawn. Animals were humanely sacrificed using an overdose of chloral hydrate and their brains harvested at indicated time points for the various analyses.

\section{Tissue preparation and immunohistochemistry}

As described $[4,26]$ rat brain tissues (40 $\mu$ m thickness) were rinsed in PBS and then, incubated with the following primary antibodies: mouse anti-neuron-specific nuclear protein (NeuN; 1:1000; Merck millipore) for general neurons, and mouse antiOX-42 (1:400; Bio-rad), which recognizes complement receptor 3; mouse anti-ED1 (1:5000; abcam), which recognizes specific for glycosylated lysosomal antigen for microglia; rabbit anti-GFAP (1:50000; Neuromics) for astrocyte. The next day, for light microscopy, tissue sections were rinsed and incubated with the appropriate biotinylated mouse (1:400; KPL, MD, USA) or rabbit (1:400, KPL, MD, USA) secondary antibody and followed by avidinbiotin-peroxidase complex (Vectastain ABC kit; Vector Laboratories, Burlingame, CA, USA) for $1 \mathrm{hr}$ at room temperature. The bound antiserum was visualized by incubating with 3,3' diaminobenzidine (DAB: Sigma) solution under a bright-field microscope (Olympus Optical, Tokyo, Japan).

For immunofluorescence staining, tissue sections were incubated overnight at RT in a combination of following primary antibodies: mouse anti-OX-42, mouse anti-GFAP (1:500, Sigma), mouse antiNeuN, goat anti-IL-13, rabbit anti-CDNF (1:400, Abnova), rabbit anti-CNTF (1:400, Santa-Cruz). After washing in PBS containing $0.5 \% \mathrm{BSA}$, the sections were incubated simultaneously with a mixture of following secondary antibodies for $1 \mathrm{hr}$ at RT. Alexa Fluor 594-conjugated anti-goat $\operatorname{IgG}(1: 500$, Invitrogen) with a Fluorescein conjugated anti-mouse $\operatorname{IgG}(1: 500$, Invitrogen); Cy3-conjugated anti-rabbit $\operatorname{IgG}(1: 1000$, Sigma) with a Fluorescein conjugated anti-mouse IgG. The stained tissue sections were viewed under confocal laser-scanning microscope (Carl Zeiss, Germany).

For Nissl staining, $40 \mu \mathrm{m}$ thick coronal sections of the striatal regions of the brain were mounted on Amininosian coated slides, dried for $1 \mathrm{hr}$ at RT, dehydrated in 100\% ethanol, cleared in xylene, hydrated in decreasing alcohol gradient, stained with $0.5 \%$ cresyl violet (Sigma), washed in distilled water, dehydrated in $100 \%$ ethanol, coverslipped, and then viewed under a bright-field microscope (Olympus Optical). 


\section{Striatal cell counting}

Tissue sections (40-um-thick coronal sections) were collected in six separate series and selected 4 evenly spaced sections from anterior to posterior in the striatum region. Cell counts and evaluation of immunoreactivity were then conducted using Adobe Photoshop CS6. Every selected section passed through the striatum region, containing up to $4.6 \times 10^{5} \mu \mathrm{m}^{2}$ of the striatum for DAB staining. Finally, immunopositive cells were counted using the "count tool" under the Analysis menu.

\section{Image J analysis}

Imaging data obtained from bright-field microscope and confocal microscope were analyzed as pixel values using the Image J program (National Institutes of Health, Washington County, MD, USA). Image J was used to quantify the chromogenic signal intensity of images adjusted above the threshold to rule out unspecific background signal. For measurement of IL-13, CDNF, CNTF, FITC-labeled albumin, images were transformed to 8-bit grayscale and adjusted at the endpoint of threshold histogram. Then the pixel value was quantified and normalized by unstained area. For measurement of IL-13 within OX-42 $2^{+}$microglia/macrophages, and CDNF or CNTF within $\mathrm{GFAP}^{+}$astrocytes, GFAP(-) area and leakage of FITC-labeled albumin from blood vessels, the adjusted images of each channel are colocalized using a colocalization plugin, then the overlaid signal are quantified.

\section{FITC-labeled albumin assay}

As previously described [14] with some modifications, a fluorescein isothiocyanate (FITC)-labeled albumin (Sigma) assay was carried out in order to analyze damage of BBB. Animals were anesthetized with chloral hydrate and killed after LPS or PBS injections. In all animals, heparin $(10 \mathrm{U} / \mathrm{ml}$ in Hank's Balanced Salt Solution) was injected into common carotid artery following cardiac puncture. Immediately after heparin was injected, $10 \mathrm{ml}$ FITC-linked albumin ( $5 \mathrm{mg} / \mathrm{ml}$, in $0.1 \mathrm{M}$ PBS buffer) was similarly infused at a rate of $1.5 \mathrm{ml} / \mathrm{min}$. Within $2 \mathrm{~min}$, the brains were removed and immersed immediately into a $4 \%$ paraformaldehyde solution (dissolved in $0.1 \mathrm{M} \mathrm{PBS}$ ) at $4{ }^{\circ} \mathrm{C}$ for 1 day; then, the brains were cryoprotected in $30 \%$ sucrose. The brains were cut on a sliding microtome in 40- $\mu \mathrm{m}$-thick coronal sections. The sections were collected, floated in 0.1 M PBS, and mounted on gelatin-subbed glass slides. The sections were dried and coverslipped using Vectashield mounting medium (Vector Laboratories). To quantify the total area of FITC-linked albumin leakage, two images of striatal regions were obtained, thresholded using Image J, quantified, and normalized by value obtained in PBS injected striatum.

\section{Statistical analysis}

Statistical analyses were performed using GraphPad Prism 5 (GraphPad Software., San Diego, CA, USA). Results are expressed as mean \pm standard error of the mean (SEM). For statistical evaluation between two groups, $\mathrm{p}$ value was assessed by Student's unpaired t-test. For comparison of multiple groups, $\mathrm{p}$ value was assessed by one-way of variance (ANOVA) with Newman-Keuls analysis. $\mathrm{p}<0.05$ was considered to indicate statistical significance.

\section{RESULTS}

\section{Interleukin 13 on microglia contributes to neurodegeneration of LPS-injected rat striatum in vivo}

LPS $(5 \mu \mathrm{g})$ or PBS as a control was unilaterally microinjected into the striatum of rats. Seven days later, brains were removed, and coronal sections were processed for neuronal nuclei (NeuN) immunohistochemical staining to detect general neurons and Nissl staining on the same sections. In the striatum treated with LPS, we observed dramatic reductions in the number of $\mathrm{NeuN}^{+} / \mathrm{Nissl}^{+}$ cells (Fig. 1C, F) compared with PBS-treated striatum (Fig. 1A, F).
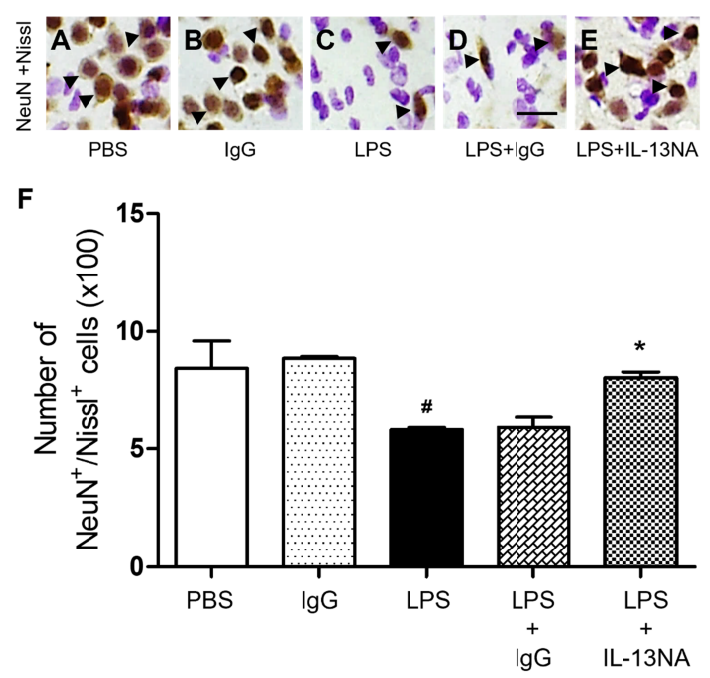

Fig. 1. Interleukin-13 contributes to neurodegeneration of LPS-injected rat striatum in vivo. Animals unilaterally received intrastriatal injection of PBS (A, $3 \mu \mathrm{l})$ or non-specific IgG (IgG) only (B, $1 \mu \mathrm{g})$ as a control, LPS (C, $5 \mu \mathrm{g}), \mathrm{LPS}+\operatorname{IgG}(\mathrm{D}, 1 \mu \mathrm{g})$ and LPS+Interleukin 13 neutralizing antibody (IL-13NA) (E, $1 \mu \mathrm{g})$. (A E) At 7 days after LPS injection, animals were sacrificed, and the coronal sections $(40 \mu \mathrm{m})$ were selected and processed for neuronal nuclei (NeuN) immunohistochemical staining and Nissl staining. Arrowheads indicate $\mathrm{NeuN}^{+}$cells merged with Nissl ${ }^{+}$cells $\left(\mathrm{NeuN}^{+} /\right.$ $\mathrm{Nissl}^{+}$). (F) Number of $\mathrm{NeuN}^{+} / \mathrm{Niss}^{+}$cells in the striatum at 7 days after LPS injection. ${ }^{*} \mathrm{p}<0.001$, as compared with PBS, ${ }^{*} \mathrm{p}<0.001$, as compared with LPS+IgG. One-way ANOVA and Newman-Keuls analyses. Four to eleven animals were used for each experimental group. The results represent mean \pm SEM. Scale bar, (A E) $25 \mu \mathrm{m}$. 
Moreover, PBS-treated striatum had a clearly defined nucleus and prominent Nissl substances (Fig. 1A) when compared with LPStreated striatum, exhibiting a significant loss of Nissl substances with gliosis (Fig. 1C). These observations indicated that $\mathrm{NeuN}^{+}$ neurons in the striatum were substantially destructed.

Accumulating evidence, including ours [14] have shown that LPS-activated microglia produce IL-13, contributing to neurodegeneration in vivo. Accordingly, we determined the activation of microglia in the striatum by LPS in vivo. Sections adjacent to those used in Fig. 1 were processed for immunohistochemical staining using antibodies against OX-42 and ED1 to detect microglial activation. In PBS-treated striatum, the majority of $\mathrm{OX}-42^{+} \mathrm{mi}-$ croglia exhibited resting morphology, specifically cell bodies and thin, long, or ramified processes (Fig. 2A). In marked contrast and consistent with our recent report [14], LPS triggered profound activation of microglia with activated morphology (larger cell bodies with short, thick, or no processes) in $\mathrm{OX}-42^{+}$cells (Fig. 2B). The

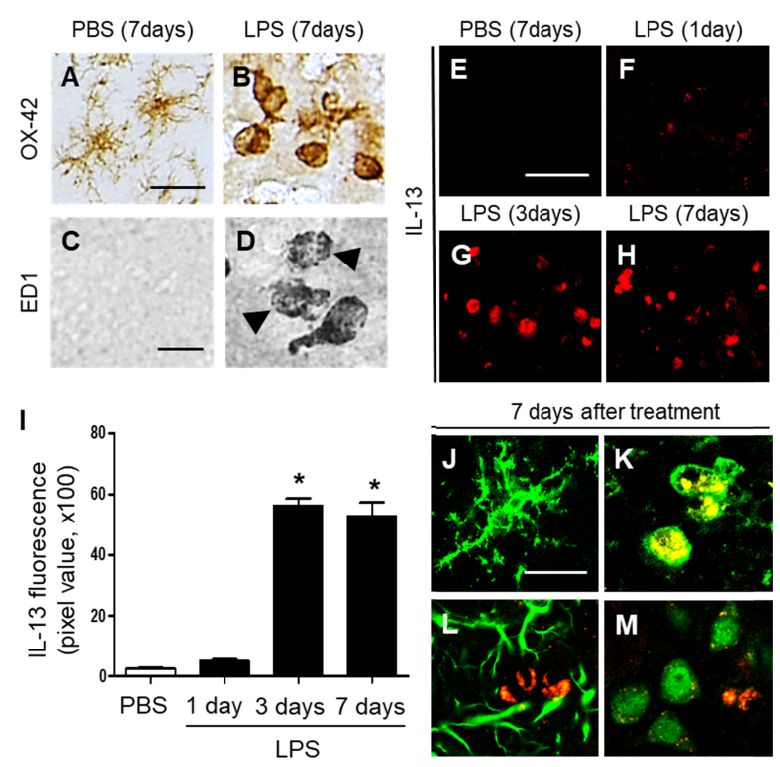

Fig. 2. Interleukin-13 expression in the striatum of LPS-injected rat in vivo. (A D) Striatal sections (A, C, PBS; B, D, LPS) adjacent to those used in Fig. $1 \mathrm{~A}$ and $\mathrm{C}$ were immunostained with OX-42 (A, B) and ED1 (C, D) antibodies for microglia. Accumulating intracellular vacuoles are denoted by arrowheads in D. (E H) IL-13 immunofluorescence staining in the striatum at 7 days after intrastriatal injection of PBS as a control (E), and at 1 day $(\mathrm{F}), 3$ days $(\mathrm{G})$, or 7 days $(\mathrm{H})$ after intrastriatal injection of LPS. (I) Quantification of IL- 13 expression in OX- $42^{+}$cells in the striatum at indicated time points. ${ }^{*} \mathrm{p}<0.001$, as compared with PBS. One-way ANOVA and Newman-Keuls analyses. Seven animals were used for each experimental group. The results represent mean \pm SEM. (J M) Immunofluorescence images of interleukin 13 (IL-13, K; red) and OX-42 (J, K; green), or IL-13 (L; red) and GFAP (L; green), or IL-13 (M; red) and NeuN (M; green) and both images are merged (Yellow; $\mathrm{K})$ in the striatum at 7 days after PBS (J) or LPS (K M) injection. Scale bars, (A, B) $25 \mu \mathrm{m}$; (C, D) 20 $\mu \mathrm{m}$; (E H) $50 \mu \mathrm{m}$; (J M) $20 \mu \mathrm{m}$. data also showed that microglia appeared to reach a state like that of active phagocytes (Fig. 2D), as determined by ED1 immunohistochemical staining, which labels phagocytic microglia, in particular, the presence of accumulating intracellular lipid vacuoles [27]. $\mathrm{ED}^{+}$cells were undetectable in the striatum on the PBS-treated control (Fig. 2C).

Next, we investigated whether LPS-activated microglia could produce IL-13 in the striatum. LPS or PBS as a control was injected into the striatum and striatal sections were processed for immunofluorescence for IL-13 at indicated time points. There was no substantial increase in IL-13 expression at 1 day post LPS (Fig. 2F, I), compared to PBS-injected control (Fig. 2E, I). By contrast, dramatic increases in IL-13 $3^{+}$cells were observed as early as 3 days after LPS injection (Fig. 2G, I), which was maintained up to 7 days after LPS injection (Fig. 2H, I), compared to PBS-injected control (Fig. 2E, I).

Expression of IL-13 was analyzed in OX- $42^{+}$microglia, GFAP ${ }^{+}$ astrocytes and $\mathrm{NeuN}^{+}$neurons at 7 days post LPS. Double immunofluorescence staining showed that in LPS-injected striatum, IL13 was merged exclusively within $\mathrm{OX}-42^{+}$microglia (Fig. $2 \mathrm{~K}$ ), but not in $\mathrm{GFAP}^{+}$astrocytes (Fig. 2L) or NeuN ${ }^{+}$neurons (Fig. 2M). In PBS-injected striatum as a control, IL-13 was undetectable in OX$42^{+}$resting microglia (Fig. $2 \mathrm{~J}$ ).

To explore the role of IL-13 in LPS-induced neurodegeneration in vivo, we examined whether IL-13 neutralizing antibody (IL13NA) altered LPS-induced neuronal damage in the striatum. NeuN immunohistochemistry and Nissl staining were performed 7 days after intrastriatal co-injection with LPS and IL-13NA. The results of $\mathrm{NeuN}^{+} / \mathrm{Nissl}^{+}$staining revealed that the combination of IL-13NA and LPS partially protected neurons in the striatum against LPS-induced neurotoxicity (Fig. 1E) compared to LPS and IgG-treated control (Fig. 1D). Data were quantified and expressed as the number of $\mathrm{NeuN}^{+} / \mathrm{Niss}^{+}$cells, in LPS-injected and IL13NA-treated striatum, the number of $\mathrm{NeuN}^{+} / \mathrm{Niss}^{+}$cells were significantly increased compared to LPS-injected and IgG-treated striatum as a control (Fig. 1F). Like PBS alone (Fig. 1A, F; Fig. 3A, B, and $\mathrm{M}$ ), the injection of non-specific IgG (Fig. 1B, F) or IL-13NA alone (Fig. 3G, H, and $\mathrm{M}$ ) as a control did not affect the number of $\mathrm{NeuN}^{+} / \mathrm{Niss}^{+}$cells in the striatum. Taken together, results indicate that IL-13 contributes to neurodegeneration in the LPS-injected rat striatum in vivo.

\section{IL-13 contributes to disruption of both $B B B$ and astrocytes in the striatum in vivo}

As IL-13 regulates blood retina barrier (BRB) integrity altered by subretinal transplantation of IL-13 expressing stem cells [19] we wonder if IL-13 produced by microglia can affect BBB permeabil- 

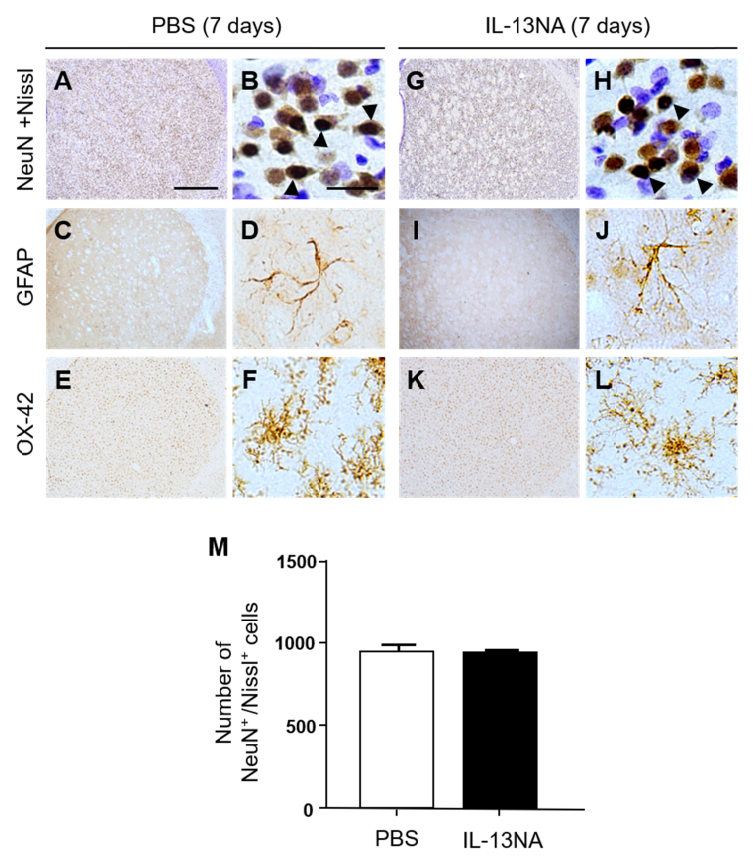

Fig. 3. Interleukin-13 neutralizing antibody has no effects on neurotoxicity and inflammation in rat striatum in vivo. Animals unilaterally received intrastriatal injection of PBS (A F, $3 \mu \mathrm{l})$ or Interleukin 13 neutralizing antibody (IL-13NA, $1 \mu \mathrm{g} ; \mathrm{G} \sim \mathrm{L}$ ) as a control. 7 days later, animals were sacrificed, and the coronal sections $(40 \mu \mathrm{m})$ were selected and processed for immunostaining with neuronal nuclei (NeuN) and Nissl staining (A, B, G, H), GFAP (C, D, I, J) and OX-42 (E, F, K, L). Arrowheads indicate NeuN ${ }^{+}$ cells merged with $\mathrm{Nissl}^{+}$cells $\left(\mathrm{NeuN}^{+} / \mathrm{Niss}^{+}\right)$. (M) Number of $\mathrm{NeuN}^{+} /$ $\mathrm{Niss}^{+}$cells in the striatum at 7 days after injection. Three animals were used for each experimental group. The results represent mean \pm SEM. Scale bar, (A, C, E, G, I, K) $100 \mu \mathrm{m}$; (B, D, F, H, J, L) $25 \mu \mathrm{m}$.

ity in the LPS-lesioned rat striatum. To test this, PBS as a control or LPS+IL-13NA or LPS+IgG (control for IL-13NA) was unilaterally injected into the rat striatum and $\mathrm{BBB}$ disruption was evaluated by detecting FITC-labeled albumin at 3 days after injection. In PBS injected rats, FITC labeled albumin was confined to the blood vessels in the striatum, indicating that the BBB was intact (Fig. 4A, D). However, the diffusion of FITC-labeled albumin from multiple blood vessels was clear and detected at 3 days in the striatum of LPS+IgG-injected rats (Fig. 4B, D). Pharmacological inhibition of IL-13 functions by IL-13NA partly blocked LPS-induced BBB damage as analyzed by the diffusion of FITC-labeled albumin (Fig. $4 \mathrm{C}, \mathrm{D})$.

Next, we wondered if direct injection of exogenous IL-13 could induce BBB damage and neurotoxicity. Two doses of rat recombinant IL-13 (50 ng and $300 \mathrm{ng}$ ) and PBS as a control was unilaterally injected into rat striatum (Fig. 5) [28, 29]. Consistent with PBS-injected striatum (Fig. 5A, D; Fig. 4A, D), FITC labeled albumin was enclosed to the blood vessels in the striatum at 3 days post IL-13 (Fig. 5B D), indicating that the BBB was intact. Ad-
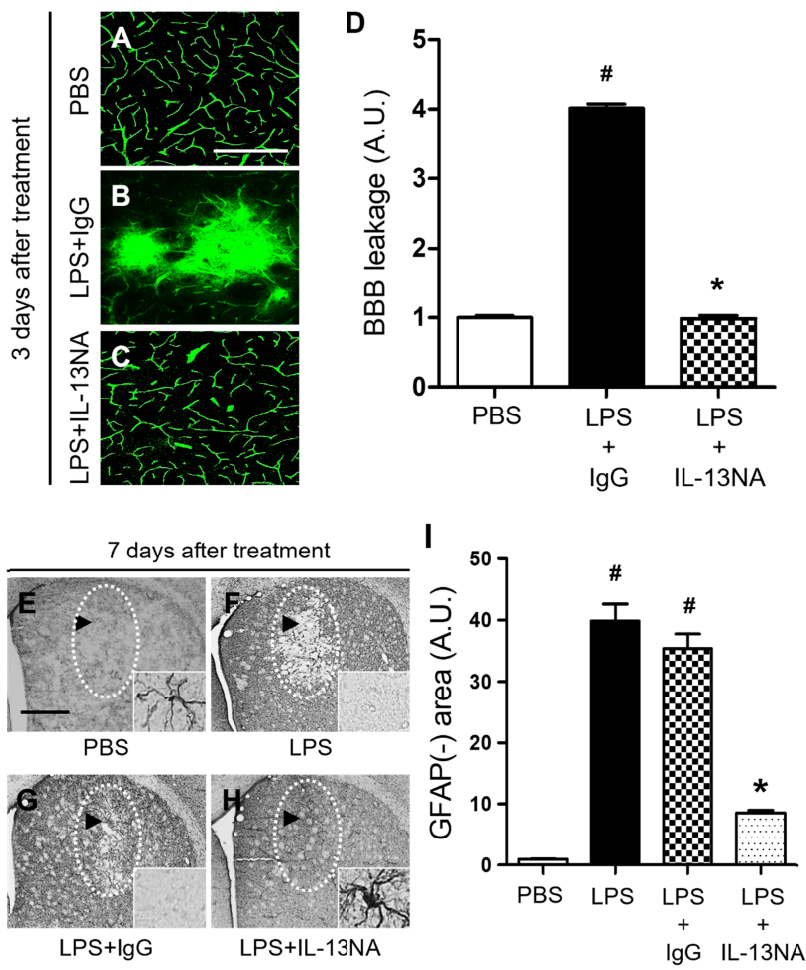

Fig. 4. Interleukin-13 contributes to disruption of BBB and loss of astrocytes and in LPS-injected rat striatum in vivo. (A C) Animals unilaterally received intrastriatal injection of PBS as a control (A) or LPS+ $\operatorname{lgG}(\mathrm{B})$ or IL-13NA (C). 3 days later, FITC-labeled albumin was administered to detect focal leakage of the BBB. (D) Quantification of BBB leakage density. ${ }^{*} \mathrm{p}<0.001$, as compared with PBS, ${ }^{*} \mathrm{p}<0.001$, as compared with LPS $+\operatorname{IgG}$. One-way ANOVA and Newman-Keuls analyses. Three to four animals were used for each experimental group. The results represent mean \pm SEM. $(\mathrm{E} \sim \mathrm{H})$ Animals unilaterally received intrastriatal injection of PBS as a control (E) or LPS only (F) or LPS+IgG (G) or LPS+IL-13NA (H). 3 days later, striatal tissues were immunostained with anti-GFAP antibody for astrocyte. Dotted round indicates area lacking in GFAP ${ }^{+}$astrocytes. Arrow indicates syringe track. (I) Quantification of GFAP immunonegative (GFAP) area at 7 days after LPS injection. $\# \mathrm{p}<0.001$, as compared with PBS, ${ }^{*} \mathrm{p}<0.001$, as compared with LPS+IgG. One-way ANOVA and Newman-Keuls analyses. Four animals were used for each experimental group. The results represent mean \pm SEM. Scale bars, (A C) $500 \mu \mathrm{m}$; $(\mathrm{E} \sim \mathrm{H})$ $100 \mu \mathrm{m}$.

ditional experiments were performed to examine neurotoxicity of exogenous IL-13. Consistent with PBS-injected striatum (Fig. 5E, H; Fig. 1A, F), NeuN immunohistochemical staining and Nissl staining showed a clearly defined nucleus and prominent Nissl substances in the striatum at 7 days post IL-13 (Fig. 5F H), indicating that exogenous IL-13 had no neurotoxicity. Taken together, these observations indicate that compared to endogenous IL-13 originated from OX-42 $2^{+}$microglia/macrophages, exogenous IL-13 fails to produce BBB damage and neurotoxicity. The discrepancies between these two experiments are probably because compared to LPS-induced endogenous IL-13, doses of exogenous IL-13 directly 


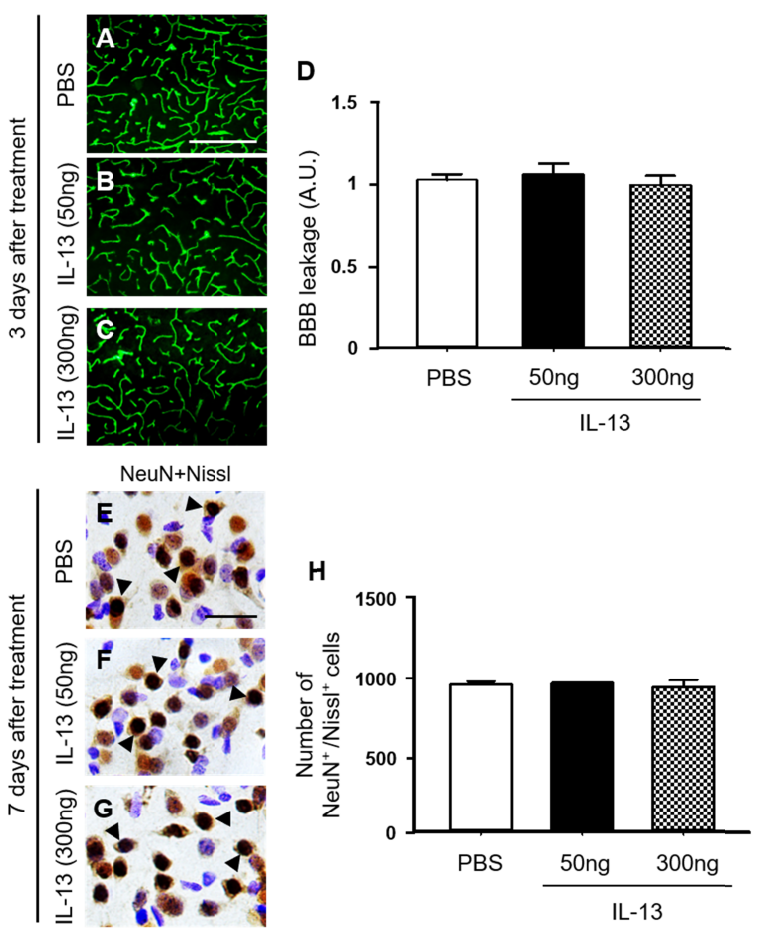

Fig. 5. Interleukin-13 has no effects on BBB damage and neurotoxicity in rat striatum in vivo. (A C) Animals unilaterally received intrastriatal injection of PBS as a control (A) or IL-13 (B, 50 ng) or IL-13 (C, $300 \mathrm{ng}$ ). 3 days later, FITC-labeled albumin was administered to detect focal leakage of the BBB. (D) Quantification of BBB leakage density. Three to four animals were used for each experimental group. The results represent mean \pm SEM. Scale bars, $(A \sim C) 500 \mu \mathrm{m}$. (E H) Animals unilaterally received intrastriatal injection of PBS as a control (E) or IL-13 (F, $50 \mathrm{ng}$ ), IL13 (G, $300 \mathrm{ng}$ ). 7 days later, striatal tissues were selected and processed for immunostaining with neuronal nuclei (NeuN) and Nissl staining $(\mathrm{E} \sim \mathrm{G})$. Arrowheads indicate $\mathrm{NeuN}^{+}$cells merged with $\mathrm{Niss}^{+}$cells $\left(\mathrm{NeuN}^{+} / \mathrm{Nisl}^{+}\right)$. (H) Number of $\mathrm{NeuN}^{+} / \mathrm{Nissl}^{+}$cells in the striatum at 7 days after injection. Three animals were used for each experimental group. The results represent mean \pm SEM. Scale bar, (A C) $500 \mu \mathrm{m}$; (E G) $25 \mu \mathrm{m}$.

injected here are not enough to produce similar BBB damage and neurotoxicity. Alternatively, microenvironments induced by LPS injection might be different from those induced by simple injection of exogenous IL-13, suggesting that LPS-induced unknown factors with endogenous IL-13 are necessary to cause BBB damage and neurotoxicity. Further studies are required to determine the discrepancy between endogenous and exogenous IL-13.

As astrocytes are closely associated with maintenance and formation of BBB [9], we wonder if IL-13 could influence astrocytes in the LPS-treated rat striatum. Immunohistochemical analysis demonstrated that in PBS-treated striatum, $\mathrm{GFAP}^{+}$cells displayed ramified morphology as a resting astrocyte (Fig. 4E, I). By contrast, areas lacking of $\mathrm{GFAP}^{+}$cells [GFAP(-) area] were observed in striatum at 7 days post LPS (Fig. 4F, I) compared to PBS-treated striatum as a control. Inhibition of IL-13 function by IL-13NA significantly reduced the GFAP(-) area in LPS-injected striatum (Fig. 4H, I) compared with LPS+IgG-treated striatum as a control (Fig. 4G, I). Like PBS (Fig. 3C, D), IL-13NA alone (Fig. 3I, J) had no effects on activation of $\mathrm{GFAP}^{+}$astrocytes. In addition, IL-13NA alone (Fig. 3K, L) also had no effects on OX-42 ${ }^{+}$microglia/macrophage, compared to PBS (Fig. 3E, F).

\section{Interleukin 13 inhibits expression of neurotrophic factors on astrocytes in LPS-injected rat striatum in vivo.}

Astrocyte is known to be involved in neuronal survival by expressing neurotrophic factors (NTFs) [5, 30,31]. Accordingly, we hypothesized that astrocytes rescued by treatment with IL-13NA (Fig. 4E I) could contribute to neuronal survival by producing neurotrophic factors. To test this, sections adjacent to those used in Fig. 3 were immunostained with GFAP antibody for astrocytes, and CDNF or CNTF antibodies to detect expression of endogenous neurotrophic factors on astrocytes. In PBS-treated striatum, the majority of $\mathrm{GFAP}^{+}$cells as a resting astrocyte did not express both CDNF (Fig. 6A, G) and CNTF (Fig. 6D, H). In LPS+IgGtreated striatum, few of $\mathrm{GFAP}^{+}, \mathrm{CDNF}^{+}$and $\mathrm{CNTF}^{+}$cells were detectable because of disruption of astrocytes (Fig. 6B, E, G, and $\mathrm{H})$. By contrast, accompanying increase in survival of $\mathrm{GFAP}^{+}$astrocytes, IL-13NA increased expression of CDNF (Fig. 6C, G) and CNTF (Fig. 6F, H) on astrocytes at 7 days post LPS.

\section{LPS-induced neurotoxicity and inflammation has no effects on degeneration of dopamine fibers in the striatum in vivo}

Next, we examined whether LPS could produce degeneration of tyrosine hydroxylase $(\mathrm{TH})^{+}$dopamine fibers and prolonged inflammation in striatum. TH immunohistochemical analysis showed that intrastriatal injection of LPS failed to induce degeneration of $\mathrm{TH}^{+}$dopamine fibers in striatum at 1 week post LPS and up to 8 weeks post LPS compared to PBS as a control (Fig. 7A C, $\mathrm{M})$, whereas LPS induced loss of striatal neurons as evidenced by NeuN immunostaining and Nissl staining (Fig. 7D F, N). OX-42 and GFAP immunohistochemical analysis showed that activation of OX-42 $2^{+}$microglia and loss of $\mathrm{GFPA}^{+}$astrocytes observed at 1 week post LPS compared to PBS control returned normal at 8 weeks post LPS (Fig. 7G L).

\section{DISCUSSION}

The present study demonstrates that LPS significantly decreases the number of $\mathrm{NeuN}^{+} / \mathrm{Niss}^{+}$cells, upregulates the expression of IL-13 exclusively within OX- $42^{+}$reactive microglia/macrophage and causes disruption of BBB in the striatum. Treatment with IL- 

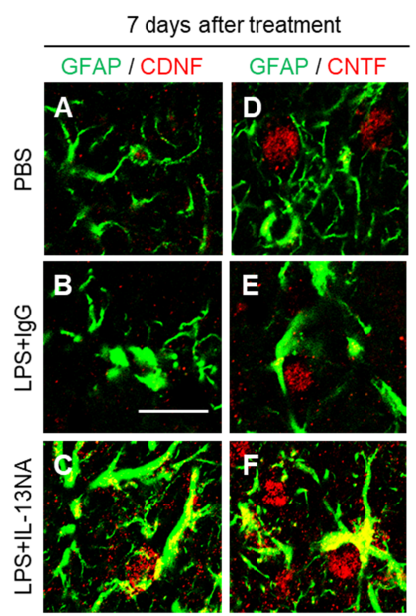
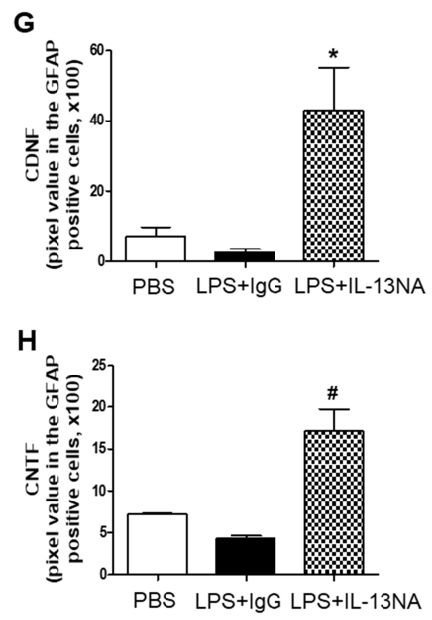

Fig. 6. Interleukin-13 inhibits expression of neurotrophic factors on astrocytes in LPS-injected rat striatum in vivo. Sections (A, D, PBS; B, E, LPS+IgG; C, F, LPS+IL-13NA) adjacent to those used in Figure 3 were immunostained with GFAP $(\mathrm{A} \sim \mathrm{F})$ antibody for astrocytes, and cerebral dopamine neurotrophic factor (CDNF: A C) or ciliary neurotrophic factor (CNTF: D F) antibodies. (A C) Immunofluorescence images of GFAP (green) and CDNF (red), and both images are merged (Yellow) in the striatum at 7 days after LPS+IgG injection. (D F) Immunofluorescence images of GFAP (green) and CNTF (red), and both images are merged (Yellow) in the striatum at 7 days after LPS+IgG injection. (G) Quantification of CDNF expression in $\mathrm{GFAP}^{+}$cells in the striatum at 7 days after LPS injection. ${ }^{*} \mathrm{p}<0.05$, as compared with LPS+IgG. One-way ANOVA and Newman-Keuls analyses. Three animals were used for each experimental group. The results represent mean \pm SEM. (H) Quantification of CNTF expression in GFAP $^{+}$cells in the striatum at 7 days after LPS+IgG injection. ${ }^{*} \mathrm{p}<0.01$, as compared with LPS+IgG. One-way ANOVA and Newman-Keuls analyses. Three animals were used for each experimental group. The results represent mean \pm SEM. Scale bar, (A F) $20 \mu \mathrm{m}$.

13NA increased the number of $\mathrm{NeuN}^{+} / \mathrm{Niss}^{+}$cells and maintained BBB integrity in LPS-injected striatum. In addition, IL-13NA blocked LPS-induced loss of GFAP ${ }^{+}$astrocytes and contributed to production of neurotrophic factors in survived astrocytes. Taken together, these data support the hypothesis that IL-13 neurotoxicity is associated with reactive microglia/macrophage, disruption of $\mathrm{BBB}$ and loss of $\mathrm{GFAP}^{+}$astrocytes in LPS-injected striatum in vivo. As reactive microglia/macrophage can produce neurotoxic molecules such as proinflammatory cytokines and reactive oxygen/ nitrosative species $[4,25]$, they are considered as one of major potential contributors to neuronal loss in vivo and in vitro $[2,32$, 33]. Here, we show neurodegeneration and activation of microglia/macrophage in LPS-injected striatum in vivo. Moreover, LPSinduced IL-13 expression was observed exclusively within reactive microglia/macrophage and IL-13 NA prevented LPS-induced degeneration of striatal neurons (Fig. 1 and 2). It is therefore likely that IL-13 might contribute to neuronal death in LPS-injected striatum by regulating reactive microglia/macrophage. This inter-
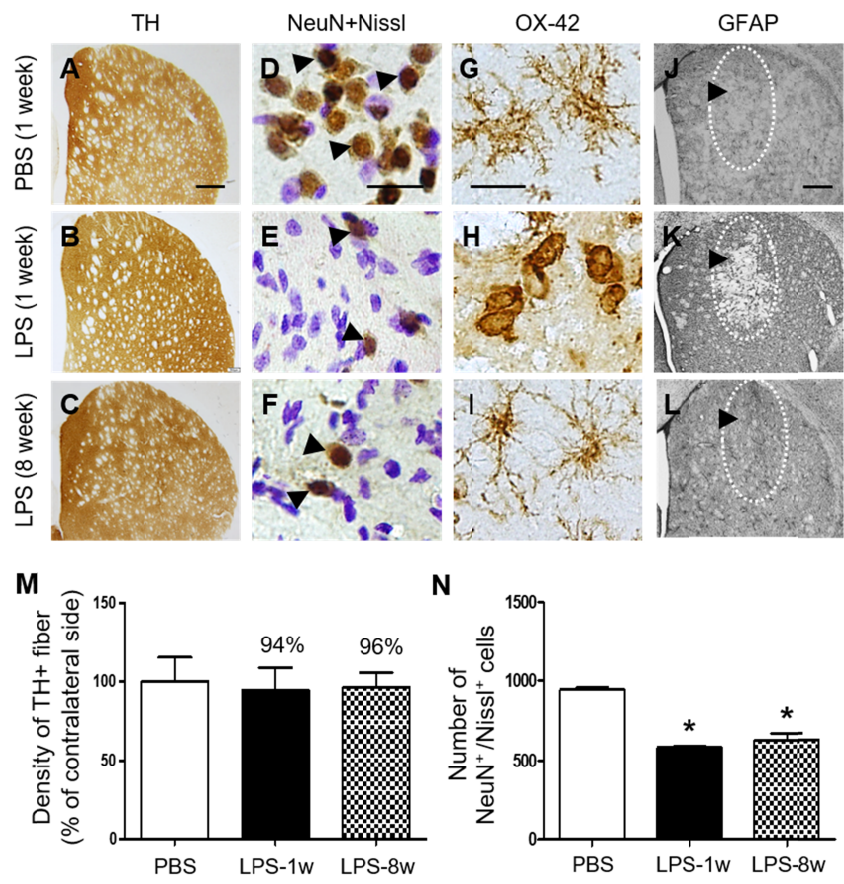

Fig. 7. Temporal effects of LPS on dopamine fibers and inflammation in the striatum in vivo. Animals unilaterally received intrastriatal injection of PBS (A, D, G, J, $3 \mu \mathrm{l}$ ) as a control and LPS (B, C, E, F, H, I, K, L, $5 \mu \mathrm{g} / 3 \mu \mathrm{l}$ ). (A C) At 1 week after LPS injection, animals were sacrificed and the coronal sections $(40 \mu \mathrm{m})$ were selected and processed for tyrosine hydroxylase (TH) immunohistochemical staining, (D F) neuronal nuclei (NeuN) immunohistochemical staining and Nissl staining, (G I) OX-42 immunohistochemical staining (insert images are ED1 Immunohistochemical staining) and (J L) GFAP immunohistochemical staining. (D F) Arrowheads indicate NeuN ${ }^{+}$cells merged with $\mathrm{Nissl}^{+}$cells $\left(\mathrm{NeuN}^{+} / \mathrm{Nissl}^{+}\right)$. (J L) Dotted round indicates area lacking in $\mathrm{GFAP}^{+}$astrocytes. Arrow indicates syringe track. (M) Density of $\mathrm{TH}^{+}$fibers in the striatum at 1 week after intrastriatal injection of PBS as a control (A) or at 1 week (B) and 8 weeks (C) after intrastriatal injection of LPS. Density of striatal $\mathrm{TH}^{+}$fibers was not significantly affected by LPS in rat striatum. One-way ANOVA and Newman-Keuls analyses. Three animals were used for each experimental group. The results represent mean \pm SEM. (N) Number of $\mathrm{NeuN}^{+} / \mathrm{Nissl}^{+}$ cells in the striatum at 1 week after intrastriatal injection of PBS as a control (D) or at 1 week (E) and 8 weeks (F) after intrastriatal injection of LPS. ${ }^{*} \mathrm{p}<0.001$, as compared with PBS. One-way ANOVA and Newman-Keuls analyses. Four to five animals were used for each experimental group. The results represent mean \pm SEM. Scale bar, (A C) $500 \mu \mathrm{m}$; (D I) $20 \mu \mathrm{m}$; (J L) $500 \mu \mathrm{m}$.

pretation is supported by findings that IL-13 was expressed within reactive microglia/macrophage and IL-13 NA contributed to neuronal survival by suppressing activation of microglia/macrophage and/or reactive microglia/macrophage-derived oxygen/nirtosative species in the hippocampus treated with thrombin [34], $\mathrm{A} \beta_{1-42}[20]$ and $\mathrm{pKr}-2$ [4].

LPS-induced neuroinflammation is known to affect BBB function by destructing the BBB integrity [35-37], which has been associated with a number of diseases, including Parkinson's disease, 
Alzheimer's disease and stroke [38-41]. BBB damage can cause infiltration of plasma components, blood cells and other neurotoxic substances into the brain parenchyma due to neuroinflammation and/or neurodegenerative processes, resulting in neuronal dysfunction and loss [42-44]. We recently demonstrated in LPS-treated rats that compromising the integrity of the $\mathrm{BBB}$ contributes to the degeneration of nigral neurons $[14,45]$. The present data show that LPS increases the filtration of FITC-linked albumin from blood vessels into striatum and IL-13 neutralization increases neuronal survival in striatum by attenuating LPS-induced damage to the BBB. It is therefore likely that IL-13 endogenously originated from microglia/macrophage may be neurotoxic by destructing BBB. Further studies will be required to determine how IL-13 can be involved in BBB damage in LPS-injected striatum while increased levels of IL-13 do not prevent neurodegeneration and BBB damage in hippocampus in a model of transient forebrain ischemia [46].

Astrocytes, the most abundant glial cells in the brain, have been implicated in maintenance and formation of BBB $[9,47]$. Bok and colleagues showed that LPS induces disruption of astrocytes and BBB damage in the substantia nigra of LPS-treated rats [14]. Astrocytes confer neuroprotection by producing neurotrophic factors (NTFs) [48]. We have recently shown that endogenous CNTF $[5,49,50]$ and CDNF (our unpublished observations) expressed on astrocytes attenuate activation of microglia/macrophage and/ or rescues nigral neurons from $\mathrm{MPP}^{+}$neurotoxicity. The present results demonstrate that LPS attenuates $\mathrm{GFAP}^{+}$astrocytes (increases GFAP(-) area) and IL-13 neutralization increases GFAP $^{+}$ astrocytes (decreases GFAP(-)area) and induces expression of CNTF and CDNF on GFAP ${ }^{+}$astrocytes. Based on these observations, we carefully suggest that CNTF and CDNF on astrocytes might be involved in rescuing LPS-induced neurotoxicity. Further studies are required to determine how IL-13 regulates expression of neurotrophic factors on astrocytes in LPS-injected striatum in vivo.

Intraperitoneal injection of LPS produced disruption of BBB in striatum and activation of astrocytes [51]. They also observed that activation of astrocytes was not associated with promoting LPSinduced disruption of BBB. This is somewhat different from our recent findings that instead of activation of astrocytes, intranigral injection of LPS induces loss of astrocytes with disruption of BBB [14]. This apparent discrepancy in experimental results between two studies is probably a result of different routes (intraperitoneal vs intranigral) used for LPS treatment. This interpretation is supported by our current findings that intrastriatal injection of LPS induces both loss of astrocytes and disruption of BBB, which is partially blocked by IL-13 neutralization. Taken together, the pres- ent data suggest that IL-13 might be involved in both damage of astrocytes and disruption of BBB in LPS-injected striatum in vivo. IL-13 is the well-known anti-inflammatory cytokine, possibly leading to maintenance of physiologic homeostasis, cell survival and tissue repair [52-54]. Exogenous human recombinant IL-13 has been shown to inhibit the development of experimental autoimmune disease and expression of pro-inflammatory cytokines in a demyelinating disease model of central nervous system [55]. Taj and colleagues [15] have reported that intracerebral delivery of IL-13 markedly decreases pro-inflammatory cytokine secretion, reduces inflammatory cell infiltration and suppresses axonal damage. In an experimental model of sepsis, endogenous IL-13 contributed to survival in mice by suppressing the excessive production of pro-inflammatory cytokines and chemokines [56]. IL13 might be related with neuroprotection in hippocampus against ischemic damage [57]. In addition, endogenous IL-13 controls brain inflammation by inhibiting expression of pro-inflammatory cytokines in LPS-treated rat cerebral cortices in vivo, resulting in an enhancement of neuronal survival [22]. These results collectively support the beneficial effects of IL-13 as an anti-inflammatory cytokine.

Conversely, harmful effects of IL-13 have also reported. An earlier study showed that IL-13 knockout mice display decreased numbers of infiltrating cells (CD11b+) and MHC-II expression in an experimental autoimmune encephalomyelitis (EAE), resulting in reduced EAE incidence [58]. Further studies on a mouse asthma model disclosed that pharmacological inhibition of IL13 by an anti-IL-13 monoclonal antibody prevents progression of inflammation by attenuating the expression of pro-inflammatory cytokines and chemokines $[59,60]$. All of these observations clearly indicate toxicity of IL-13, which is line with our present findings that IL-13 neutralization protects striatal neurons from LPS neurotoxicity in vivo.

Regarding the direct effects of IL-13 on neurons, Morrison and colleagues [21] demonstrated that expression of IL-13 receptor alphal chain (IL-13Ra1) on nigral dopamine neurons increased their susceptibility to LPS-induced oxidative stress and contributed to neurodegeneration, indicating that IL-13 might be directly neurotoxic. This interpretation is supported by the finding that lack of IL-13Ral delays degeneration of nigral dopamine neurons exposed to chronic stress [61]. Despite lacking evidence in our present study, these observations cannot rule out the possibilities of the direct neurotoxicity of IL-13 in LPS-injected striatum in vivo. This direct neurotoxicity would be in addition to the indirect neurotoxicity of IL-13 due to BBB damage and loss of astrocytes on striatal neurons. However, additional studies are required to prove direct actions of endogenous IL-13 on neurons in LPS- 
injected striatum in vivo.

It seems noteworthy that the current results are in contrast with the previous findings where expression of IL-13 controls brain inflammation by producing the death of reactive microglia/macrophage, resulting in an eventual enhancement of neuronal survival in LPS-injected cortex in vivo [22]. In the present study, we demonstrated that IL-13 originated from reactive microglia/macrophage mediates destruction of BBB and loss of astrocytes, leading to neurodegeneration in LPS-injected striatum in vivo. This finding is in line with our reports showing that IL-13 contributes to the production of proinflammatory cytokines and/or oxidative stress, leading to neurodegeneration in thrombin- [34], $\mathrm{A} \beta_{1-42^{-}}[20]$ or pKr-2-injected hippocampus in vivo [4]. It is therefore likely that these apparent discrepancies may be attributed to the use of different stimuli (LPS vs thrombin, $\mathrm{A} \beta_{1-42}$ or $\mathrm{pKr}-2$ ) and/or target areas (striatum vs cortex or hippocampus) although the underlying mechanisms remain to be determined.

Finally, the present study demonstrates that single injection of total LPS (5 ug) into striatum does not cause substantial degeneration of striatal $\mathrm{TH}^{+}$fibers at 1 week and even up to 8 weeks post LPS whereas LPS-induced neurotoxicity (loss of $\mathrm{NeuN}^{+} / \mathrm{Niss}^{+}$ cells) is maintained up to 8 weeks post LPS (Fig. 7 and Fig. 1). Changes in activation of OX-42 $2^{+}$microglia/macrophages and loss of $\mathrm{GFAP}^{+}$astrocytes returned to normal at 8 weeks post LPS compared to 1 week post LPS (Fig. 7; Fig. 2 and 3). Our results suggest that single injection of low dose LPS ( $5 \mathrm{ug}$ ) might not be enough to produce degeneration of striatal $\mathrm{TH}^{+}$fibers and prolonged neuroinflammation (changes in $\mathrm{OX}-42^{+}$and $\mathrm{GFAP}^{+}$cells) although it is neurotoxic to striatal neurons. Regarding this, intrastriatal injection of LPS (total $10 \mathrm{ug}$ ) produces activation of microglia/ macrophages in the striatum with no changes in density of striatal $\mathrm{TH}^{+}$fibers [62]. By contrast, Intrastriatal administration of LPS (total $21.5 \mathrm{ug}$ ) induces reactive microglia/macrophages-driven reduction in density of $\mathrm{TH}^{+}$fibers in striatum [63]. This apparent discrepancy in experimental results between two studies is probably a result of different doses of total LPS (21.5 ug vs $10 \mathrm{ug}$ ) and/ or number of injection sites (one site vs four sites). This interpretation is supported by the findings that decrease dopamine with activation of microglia/macrophages was observed in striatum when the higher doses of LPS (16, 32 and $60 \mathrm{ug}$ ) were administered into the striatum at three sites [64]. All these observations, including ours indicate that appropriate LPS dose and multiple injection sites into striatum are required to produce degeneration of striatal $\mathrm{TH}^{+}$ fibers, which may a highly relevant LPS-induced neuroinflammation model of PD.

\section{ACKNOWLEDGEMENTS}

This research was funded by the National Research Foundation of Korea (NRF) grant by the Korean Government (NRF2019R1A2C2007897, NRF-2017M3C7A1031105, NRF2020R1C1C1014054).

\section{AUTHOR CONTRIBUTIONS}

Conceptualization, S-Y.W. and B.K.J.; data curation, Y.C.C., J.G.J., S-Y.W., A.R.H., and B.K.J.; formal analysis, Y.C.C., J.G.J., S-Y.W., A.R.H.; funding acquisition, S-Y.W and B.K.J.; investigation, Y.C.C., J.G.J., S-Y.W., and A.R.H.; project administration, B.K.J.; supervision, B.K.J.; validation, Y.C.C., J.G.J., S-Y.W., A.R.H.; writing-original draft, Y.C.C., J.G.J. S-Y.W., A.R.H., and B.K.J. All authors have read and agreed to the published version of the manuscript.

\section{CONFLICT OF INTEREST}

The authors declare no conflict of interest.

\section{REFERENCES}

1. Leng F, Edison P (2021) Neuroinflammation and microglial activation in Alzheimer disease: where do we go from here? Nat Rev Neurol 17:157-172.

2. Chung YC, Ko HW, Bok E, Park ES, Huh SH, Nam JH, Jin BK (2010) The role of neuroinflammation on the pathogenesis of Parkinson's disease. BMB Rep 43:225-232.

3. Deng I, Corrigan F, Zhai G, Zhou XF, Bobrovskaya L (2020) Lipopolysaccharide animal models of Parkinson's disease: recent progress and relevance to clinical disease. Brain Behav Immun Health 4:100060.

4. Jeong JY, Wi R, Chung YC, Jin BK (2021) Interleukin-13 propagates prothrombin kringle-2-induced neurotoxicity in hippocampi in vivo via oxidative stress. Int J Mol Sci 22:3486.

5. Nam JH, Park ES, Won SY, Lee YA, Kim KI, Jeong JY, Baek JY, Cho EJ, Jin M, Chung YC, Lee BD, Kim SH, Kim EG, Byun K, Lee B, Woo DH, Lee CJ, Kim SR, Bok E, Kim YS, Ahn TB, Ko HW, Brahmachari S, Pletinkova O, Troconso JC, Dawson VL, Dawson TM, Jin BK (2015) TRPV1 on astrocytes rescues nigral dopamine neurons in Parkinson's disease via CNTF. Brain 138(Pt 12):3610-3622.

6. Fischer D, Hauk TG, Müller A, Thanos S (2008) Crystallins of the beta/gamma-superfamily mimic the effects of lens injury and promote axon regeneration. Mol Cell Neurosci 37:471479. 
7. Shen Y, Sun A, Wang Y, Cha D, Wang H, Wang F, Feng L, Fang S, Shen Y (2012) Upregulation of mesencephalic astrocytederived neurotrophic factor in glial cells is associated with ischemia-induced glial activation. J Neuroinflammation 9:254.

8. Aly AE, Harmon BT, Padegimas L, Sesenoglu-Laird O, Cooper MJ, Waszczak BL (2019) Intranasal delivery of pGDNF DNA nanoparticles provides neuroprotection in the rat 6-hydroxydopamine model of Parkinson's disease. Mol Neurobiol 56:688-701.

9. Cabezas R, Avila M, Gonzalez J, El-Bachá RS, Báez E, GarcíaSegura LM, Jurado Coronel JC, Capani F, Cardona-Gomez GP, Barreto GE (2014) Astrocytic modulation of blood brain barrier: perspectives on Parkinson's disease. Front Cell Neurosci 8:211.

10. Linnerbauer M, Wheeler MA, Quintana FJ (2020) Astrocyte crosstalk in CNS inflammation. Neuron 108:608-622.

11. Giovannoni F, Quintana FJ (2020) The role of astrocytes in CNS inflammation. Trends Immunol 41:805-819.

12. Kim GW, Gasche Y, Grzeschik S, Copin JC, Maier CM, Chan PH (2003) Neurodegeneration in striatum induced by the mitochondrial toxin 3-nitropropionic acid: role of matrix metalloproteinase- 9 in early blood-brain barrier disruption? J Neurosci 23:8733-8742.

13. Espinosa-Oliva AM, de Pablos RM, Sarmiento M, Villarán RF, Carrillo-Jiménez A, Santiago M, Venero JL, Herrera AJ, Cano J, Machado A (2014) Role of dopamine in the recruitment of immune cells to the nigro-striatal dopaminergic structures. Neurotoxicology 41:89-101.

14. Bok E, Cho EJ, Chung ES, Shin WH, Jin BK (2018) Interleukin- 4 contributes to degeneration of dopamine neurons in the lipopolysaccharide-treated substantia nigra in vivo. Exp Neurobiol 27:309-319.

15. Hamzei Taj S, Le Blon D, Hoornaert C, Daans J, Quarta A, Praet J, Van der Linden A, Ponsaerts P, Hoehn M (2018) Targeted intracerebral delivery of the anti-inflammatory cytokine IL13 promotes alternative activation of both microglia and macrophages after stroke. J Neuroinflammation 15:174.

16. Miao W, Zhao Y, Huang Y, Chen D, Luo C, Su W, Gao Y (2020) IL-13 ameliorates neuroinflammation and promotes functional recovery after traumatic brain injury. J Immunol 204:1486-1498.

17. Mori S, Maher P, Conti B (2016) Neuroimmunology of the interleukins 13 and 4. Brain Sci 6:18.

18. Quarta A, Berneman Z, Ponsaerts P (2020) Neuroprotective modulation of microglia effector functions following priming with interleukin 4 and 13: current limitations in understand- ing their mode-of-action. Brain Behav Immun 88:856-866.

19. Huang L, You J, Yao Y, Xie M (2020) Interleukin-13 gene modification enhances grafted mesenchymal stem cells survival after subretinal transplantation. Cell Mol Neurobiol 40:725735 .

20. Nam JH, Park KW, Park ES, Lee YB, Lee HG, Baik HH, Kim YS, Maeng S, Park J, Jin BK (2012) Interleukin-13/-4-induced oxidative stress contributes to death of hippocampal neurons in aß1-42-treated hippocampus in vivo. Antioxid Redox Signal 16:1369-1383.

21. Morrison BE, Marcondes MC, Nomura DK, Sanchez-Alavez M, Sanchez-Gonzalez A, Saar I, Kim KS, Bartfai T, Maher P, Sugama S, Conti B (2012) Cutting edge: IL-13Ral expression in dopaminergic neurons contributes to their oxidative stress-mediated loss following chronic peripheral treatment with lipopolysaccharide. J Immunol 189:5498-5502.

22. Shin WH, Lee DY, Park KW, Kim SU, Yang MS, Joe EH, Jin BK (2004) Microglia expressing interleukin-13 undergo cell death and contribute to neuronal survival in vivo. Glia 46:142-152.

23. Kolosowska N, Keuters MH, Wojciechowski S, Keksa-Goldsteine V, Laine M, Malm T, Goldsteins G, Koistinaho J, Dhungana H (2019) Peripheral administration of IL-13 induces anti-inflammatory microglial/macrophage responses and provides neuroprotection in ischemic stroke. Neurotherapeutics 16:1304-1319.

24. Azimzadeh M, Mahmoodi M, Kazemi M, Hakemi MG, Jafarinia M, Eslami A, Salehi H, Amirpour N (2020) The immunoregulatory and neuroprotective effects of human adipose derived stem cells overexpressing IL-11 and IL-13 in the experimental autoimmune encephalomyelitis mice. Int Immunopharmacol 87:106808.

25. Jeong JY, Chung YC, Jin BK (2019) Interleukin-4 and interleukin-13 exacerbate neurotoxicity of prothrombin kringle-2 in cortex in vivo via oxidative stress. Int J Mol Sci 20:1927.

26. Chung YC, Jeong JY, Jin BK (2020) Interleukin-4-mediated oxidative stress is harmful to hippocampal neurons of prothrombin kringle-2-lesioned rat in vivo. Antioxidants (Basel) 9:1068.

27. Popovich PG, Wei P, Stokes BT (1997) Cellular inflammatory response after spinal cord injury in Sprague-Dawley and Lewis rats. J Comp Neurol 377:443-464.

28. Kawahara K, Suenobu M, Yoshida A, Koga K, Hyodo A, Ohtsuka H, Kuniyasu A, Tamamaki N, Sugimoto Y, Nakayama H (2012) Intracerebral microinjection of interleukin-4/interleukin-13 reduces $\beta$-amyloid accumulation in the ipsilateral side and improves cognitive deficits in young amyloid precursor 
protein 23 mice. Neuroscience 207:243-260.

29. Bluthé RM, Bristow A, Lestage J, Imbs C, Dantzer R (2001) Central injection of interleukin-13 potentiates LPS-induced sickness behavior in rats. Neuroreport 12:3979-3983.

30. Lindholm P, Voutilainen MH, Laurén J, Peränen J, Leppänen VM, Andressoo JO, Lindahl M, Janhunen S, Kalkkinen N, Timmusk T, Tuominen RK, Saarma M (2007) Novel neurotrophic factor CDNF protects and rescues midbrain dopamine neurons in vivo. Nature 448:73-77.

31. Kim KI, Baek JY, Chung YC, Nam JH, Shin WH, Jin BK (2021) p70S6K on astrocytes protects dopamine neurons from 1-methyl-4-phenylpyridinium neurotoxicity. Glia 69:21332145.

32. Afridi R, Lee WH, Suk K (2020) Microglia gone awry: linking immunometabolism to neurodegeneration. Front Cell Neurosci 14:246.

33. Aguzzi A, Zhu C (2017) Microglia in prion diseases. J Clin Invest 127:3230-3239.

34. Park KW, Baik HH, Jin BK (2009) IL-13-induced oxidative stress via microglial NADPH oxidase contributes to death of hippocampal neurons in vivo. J Immunol 183:4666-4674.

35. Wispelwey B, Lesse AJ, Hansen EJ, Scheld WM (1988) Haemophilus influenzae lipopolysaccharide-induced blood brain barrier permeability during experimental meningitis in the rat. J Clin Invest 82:1339-1346.

36. Huang X, Hussain B, Chang J (2021) Peripheral inflammation and blood-brain barrier disruption: effects and mechanisms. CNS Neurosci Ther 27:36-47.

37. Logsdon AF, Erickson MA, Chen X, Qiu J, Lim YP, Stonestreet BS, Banks WA (2020) Inter-alpha inhibitor proteins attenuate lipopolysaccharide-induced blood-brain barrier disruption and downregulate circulating interleukin 6 in mice. J Cereb Blood Flow Metab 40:1090-1102.

38. Tucsek Z, Toth P, Tarantini S, Sosnowska D, Gautam T, Warrington JP, Giles CB, Wren JD, Koller A, Ballabh P, Sonntag WE, Ungvari Z, Csiszar A (2014) Aging exacerbates obesityinduced cerebromicrovascular rarefaction, neurovascular uncoupling, and cognitive decline in mice. J Gerontol A Biol Sci Med Sci 69:1339-1352.

39. Sengillo JD, Winkler EA, Walker CT, Sullivan JS, Johnson M, Zlokovic BV (2013) Deficiency in mural vascular cells coincides with blood-brain barrier disruption in Alzheimer's disease. Brain Pathol 23:303-310.

40. Gray MT, Woulfe JM (2015) Striatal blood-brain barrier permeability in Parkinson's disease. J Cereb Blood Flow Metab 35:747-750.

41. Fujimoto T, Morofuji Y, Kovac A, Erickson MA, Deli MA,
Niwa M, Banks WA (2021) Pitavastatin ameliorates lipopolysaccharide-induced blood-brain barrier dysfunction. Biomedicines 9:837.

42. Zlokovic BV (2008) The blood-brain barrier in health and chronic neurodegenerative disorders. Neuron 57:178-201.

43. Lee CH, Ahn JH, Lee TK, Sim H, Lee JC, Park JH, Shin MC, Cho JH, Kim DW, Won MH, Choi SY (2021) Comparison of neuronal death, blood-brain barrier leakage and inflammatory cytokine expression in the hippocampal CA1 region following mild and severe transient forebrain ischemia in gerbils. Neurochem Res 46:2852-2866.

44. Farmen K, Tofiño-Vian M, Iovino F (2021) Neuronal damage and neuroinflammation, a bridge between bacterial meningitis and neurodegenerative diseases. Front Cell Neurosci 15:680858.

45. Bok E, Chung YC, Kim KS, Baik HH, Shin WH, Jin BK (2018) Modulation of M1/M2 polarization by capsaicin contributes to the survival of dopaminergic neurons in the lipopolysaccharide-lesioned substantia nigra in vivo. Exp Mol Med 50:114.

46. Lee TK, Park Y, Kim B, Lee JC, Shin MC, Ohk TG, Park CW, Cho JH, Park JH, Lee CH, Won MH, Ahn JH (2020) Longterm alternating fasting increases interleukin-13 in the gerbil hippocampus, but does not protect BBB and pyramidal neurons from ischemia-reperfusion injury. Neurochem Res 45:2352-2363.

47. Verkhratsky A, Nedergaard M (2018) Physiology of astroglia. Physiol Rev 98:239-389.

48. Udovin L, Quarracino C, Herrera MI, Capani F, Otero-Losada M, Perez-Lloret S (2020) Role of astrocytic dysfunction in the pathogenesis of Parkinson's disease animal models from a molecular signaling perspective. Neural Plast 2020:1859431.

49. Baek JY, Jeong JY, Kim KI, Won SY, Chung YC, Nam JH, Cho EJ, Ahn TB, Bok E, Shin WH, Jin BK (2018) Inhibition of microglia-derived oxidative stress by ciliary neurotrophic factor protects dopamine neurons in vivo from $\mathrm{MPP}^{+}$neurotoxicity. Int J Mol Sci 19:3543.

50. Kim KI, Baek JY, Jeong JY, Nam JH, Park ES, Bok E, Shin WH, Chung YC, Jin BK (2019) Delayed treatment of capsaicin produces partial motor recovery by enhancing dopamine function in $\mathrm{MPP}^{+}$-lesioned rats via ciliary neurotrophic factor. Exp Neurobiol 28:289-299.

51. Banks WA, Gray AM, Erickson MA, Salameh TS, Damodarasamy M, Sheibani N, Meabon JS, Wing EE, Morofuji Y, Cook DG, Reed MJ (2015) Lipopolysaccharide-induced blood-brain barrier disruption: roles of cyclooxygenase, oxidative stress, neuroinflammation, and elements of the neuro- 
vascular unit. J Neuroinflammation 12:223.

52. Van Dyken SJ, Locksley RM (2013) Interleukin-4- and interleukin-13-mediated alternatively activated macrophages: roles in homeostasis and disease. Annu Rev Immunol 31:317343.

53. Bosurgi L, Cao YG, Cabeza-Cabrerizo M, Tucci A, Hughes LD, Kong Y, Weinstein JS, Licona-Limon P, Schmid ET, Pelorosso F, Gagliani N, Craft JE, Flavell RA, Ghosh S, Rothlin CV (2017) Macrophage function in tissue repair and remodeling requires IL-4 or IL-13 with apoptotic cells. Science 356:1072-1076.

54. Zhang MZ, Wang X, Wang Y, Niu A, Wang S, Zou C, Harris RC (2017) IL-4/IL-13-mediated polarization of renal macrophages/dendritic cells to an M2a phenotype is essential for recovery from acute kidney injury. Kidney Int 91:375-386.

55. Cash E, Minty A, Ferrara P, Caput D, Fradelizi D, Rott O (1994) Macrophage-inactivating IL-13 suppresses experimental autoimmune encephalomyelitis in rats. J Immunol 153:42584267.

56. Matsukawa A, Hogaboam CM, Lukacs NW, Lincoln PM, Evanoff HL, Strieter RM, Kunkel SL (2000) Expression and contribution of endogenous IL-13 in an experimental model of sepsis. J Immunol 164:2738-2744.

57. Kim DW, Lee JC, Cho JH, Park JH, Ahn JH, Chen BH, Shin BN, Tae HJ, Seo JY, Cho JH, Kang IJ, Hong S, Kim YM, Won MH, Kim IH (2015) Neuroprotection of ischemic preconditioning is mediated by anti-inflammatory, not pro-inflammatory, cytokines in the gerbil hippocampus induced by a subsequent lethal transient cerebral ischemia. Neurochem Res 40:1984-1995.

58. Sinha S, Kaler LJ, Proctor TM, Teuscher C, Vandenbark AA, Offner H (2008) IL-13-mediated gender difference in sus- ceptibility to autoimmune encephalomyelitis. J Immunol 180:2679-2685.

59. Yang G, Volk A, Petley T, Emmell E, Giles-Komar J, Shang X, Li J, Das AM, Shealy D, Griswold DE, Li L (2004) Anti-IL-13 monoclonal antibody inhibits airway hyperresponsiveness, inflammation and airway remodeling. Cytokine 28:224-232.

60. Yang G, Li L, Volk A, Emmell E, Petley T, Giles-Komar J, Rafferty P, Lakshminarayanan M, Griswold DE, Bugelski PJ, Das AM (2005) Therapeutic dosing with anti-interleukin-13 monoclonal antibody inhibits asthma progression in mice. J Pharmacol Exp Ther 313:8-15.

61. Mori S, Sugama S, Nguyen W, Michel T, Sanna MG, SanchezAlavez M, Cintron-Colon R, Moroncini G, Kakinuma Y, Maher P, Conti B (2017) Lack of interleukin-13 receptor al delays the loss of dopaminergic neurons during chronic stress. J Neuroinflammation 14:88.

62. Hoban DB, Connaughton E, Connaughton C, Hogan G, Thornton C, Mulcahy P, Moloney TC, Dowd E (2013) Further characterisation of the LPS model of Parkinson's disease: a comparison of intra-nigral and intra-striatal lipopolysaccharide administration on motor function, microgliosis and nigrostriatal neurodegeneration in the rat. Brain Behav Immun 27:91-100

63. Choi DY, Liu M, Hunter RL, Cass WA, Pandya JD, Sullivan PG, Shin EJ, Kim HC, Gash DM, Bing G (2009) Striatal neuroinflammation promotes Parkinsonism in rats. PLoS One 4:e5482.

64. Hunter RL, Dragicevic N, Seifert K, Choi DY, Liu M, Kim HC, Cass WA, Sullivan PG, Bing G (2007) Inflammation induces mitochondrial dysfunction and dopaminergic neurodegeneration in the nigrostriatal system. J Neurochem 100:13751386. 